\title{
Correlation between the UHECRs measured by the Pierre Auger Observatory and Telescope Array and neutrino candidate events from IceCube
}

\author{
A. Christov ${ }^{1}$, G. Golup ${ }^{2}$, T. Montaruli ${ }^{1}$, M. Rameez ${ }^{1, a}$ for the IceCube Collaboration ${ }^{\text {, }}$ J. Aublin ${ }^{3}$, \\ L. Caccianiga ${ }^{3}$, P.L. Ghia ${ }^{3}$, E. Roulet ${ }^{2}$, M. Unger $^{4}$ for the Pierre Auger Collaboration ${ }^{\text {c }}$, H. Sagawa ${ }^{5}$, \\ and P. Tinyakov ${ }^{6}$ for the Telescope Array Collaboration ${ }^{\mathrm{d}}$ \\ ${ }^{1}$ Département de physique nucléaire et corpusculaire, Université de Genève, 24 Quai Ernest Ansermet, \\ 1211 Genève, Switzerland \\ ${ }^{2}$ Centro Atómico Bariloche, Av. Bustillo 9500, S. C. de Bariloche 8400, Argentina \\ ${ }^{3}$ Laboratoire de Physique Nucléaire et de Hautes Energies (LPNHE), Universités Paris 6 et Paris 7 , \\ CNRS-IN2P3, 4 place Jussieu, 75252 Paris, France \\ ${ }^{4}$ Karlsruhe Institute of Technology - Campus North - Institut fër Kernphysik, Karlsruhe, Germany \\ and New York University, New York, USA \\ ${ }^{5}$ Institute for Cosmic Ray Research, University of Tokyo, Kashiwa, Chiba, Japan \\ ${ }^{6}$ Service de Physique Théorique, Université Libre de Bruxelles, Boulevard du Triomphe (Campus de \\ la Plaine), Ixelles 1050, Belgium
}

\begin{abstract}
We present the results of three searches for correlations between ultra-high energy cosmic ray events measured by Telescope Array and the Pierre Auger Observatory and high-energy neutrino candidate events from IceCube. Two cross-correlation analyses of ultra-high energy cosmic rays are done: one with 39 "cascades" from the IceCube "high-energy starting events" sample and the other one with 16 high-energy "tracks". The angular separation between the arrival directions of neutrinos and UHECRs is scanned. The same events are also used in a separate search stacking the neutrino arrival directions and using a maximum likelihood approach. We assume that UHECR magnetic deflections are inversely proportional to the energy with values $3^{\circ}, 6^{\circ}$ and $9^{\circ}$ at $100 \mathrm{EeV}$ to account for the uncertainties in the magnetic field strength and UHECR charge. A similar analysis is performed on stacked UHECR arrival directions and the IceCube 4-year sample of throughgoing muon-track events that was optimized for neutrino point source searches.
\end{abstract}

\section{Introduction}

The sources of ultra-high energy cosmic rays (UHECRs) have so far not been identified. The CRs do not point back to their sources as they are charged particles and are deflected en-route to Earth. Since both CR composition at ultra-high energies as well as the intervening magnetic field strength are poorly

\footnotetext{
a e-mail: Mohamed.Rameez@unige.ch

b http://icecube.wisc.edu/collaboration/authors/current

${ }^{c}$ http://www.auger.org/archive/authors_2015_12.html

d http://www.telescopearray.org/index.php/research/collaborators
}

This is an Open Access article distributed under the terms of the Creative Commons Attribution License 4.0, which permits unrestricted use, distribution, and reproduction in any medium, provided the original work is properly cited. 
known, this deflection cannot be computed precisely. If the CR composition is light i.e. mainly protons, the magnetic deflection may be only a few degrees at energies above a few tens of EeV. Secondary particles including neutrinos (vs) are produced in the sources by the interactions between the CRs and ambient photon and matter fields. Neutrinos have no charge and interact only through the weak force, so their arrival directions do point back to where they originated from, even though they are hard to detect. This work describes a joint analysis by the IceCube, Pierre Auger and Telescope Array Collaborations to search for angular correlations between the arrival directions of high-energy vs and UHECRs.

\section{The observatories and data sets}

\subsection{The IceCube Neutrino Telescope}

IceCube is a cubic-kilometer neutrino detector embedded in the ice at the geographic South Pole [1] between depths of $1450 \mathrm{~m}$ and $2450 \mathrm{~m}$. Different IceCube data sets are considered in this work. The first is a set of cascades ( $v_{e}$ and $v_{\tau}$ charged-current, and all flavour neutral-current interactions) that have been detected in a search for high-energy events where the interaction occurs within the detector [2]. These 39 cascades, which are part of the HESE ("High-Energy Starting Events") set (deposited energy range: 30-2000 TeV) are derived from data taken between May 2010 and May 2014 and are hereby called "high-energy cascades". A second set of events referred to as "high-energy tracks" (energy above $\sim 70 \mathrm{TeV}$ ) consists of the 7 tracks ( $v_{\mu}$ charge current interactions) in the HESE sample [2] that have energies and directions which make them more likely to be of extraterrestrial origin than the other track events in that sample and 9 muon tracks found in a search of a diffuse up-going $v_{\mu}$ flux [3].

The third data set used is called the "4-year point-source sample" [4] and consists of tracks detected between May 2008 and May 2012 with sub-degree median angular resolution. The set includes about 400,000 events, mostly up-going atmospheric vs from the Northern hemisphere and high-energy atmospheric muons from the Southern hemisphere.

\subsection{The Pierre Auger Observatory}

The Pierre Auger Observatory, located in Malargüe, Argentina (35.2 S, 69.5 W, $1400 \mathrm{~m}$ a.s.1.) [5, 6], consists of surface detectors and air fluorescence telescopes designed to perform complementary measurements of air showers created by UHECRs. The data set used for the present analysis includes 231 events with $\mathrm{E}>52 \mathrm{EeV}$ and zenith angles smaller than $80^{\circ}$ recorded by the surface detector array from January 2004 to March 2014 [7]. The exposure determined by geometrical considerations for the period analyzed amounts to $66,452 \mathrm{~km}^{2} \mathrm{sr}$ yr. The angular resolution is better than $0.9^{\circ}$. The absolute energy scale has a systematic uncertainty of $\sim 14 \%$ and the energy resolution is $\sim 12 \%$.

\subsection{Telescope Array}

The Telescope Array (TA) is located in Utah, USA (39.3 ${ }^{\circ} \mathrm{N}, 112.9^{\circ} \mathrm{W}, 1400 \mathrm{~m}$ a.s.1.) [8] and similarly detects extensive air showers generated by UHECRs. The UHECR sample considered in the present analysis consists of 87 events with $\mathrm{E}>57 \mathrm{EeV}$ and zenith angles smaller than $55^{\circ}$ collected between May 2008 and May 2014 by the surface detector array. A subset of events has been published in [9]. The total exposure is around $9,500 \mathrm{~km}^{2} \mathrm{sr}$ yr. The angular resolution is better than $1.5^{\circ}$. As with the PAO the energy scale of the surface detector array is cross-calibrated with fluorescence telescopes. The energy resolution is better than $20 \%$ with a systematic uncertainty on the absolute energy scale of $21 \%$. 


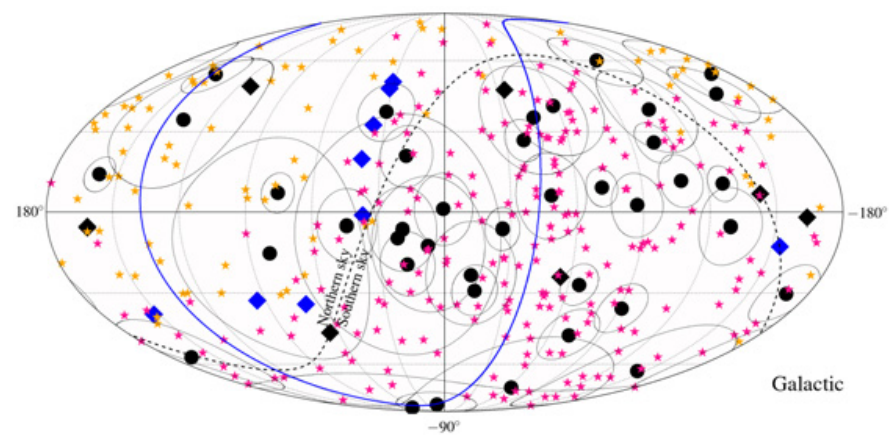

Figure 1. Map in Galactic coordinates showing the arrival directions of the IceCube cascades (black dots) and tracks (diamonds), as well as those of the UHECRs detected by the Pierre Auger Observatory (magenta stars) and Telescope Array (orange stars). The circles around the showers indicate angular errors. The black diamonds are the HESE tracks while the blue diamonds stand for the tracks from the through-going muon sample. The blue curve indicates the Super-Galactic plane.

\section{The searches}

This section describes three different analyses. A cross-correlation and a stacking likelihood analysis are done on the sample of high-energy cascades and high-energy tracks and the UHECRs detected by Auger and TA. Cascade and track-like events are considered separately since, due to their different angular resolutions, the angular distance at which a signal (if any) can be detected would be different. A third analysis is performed on stacked UHECRs and the IceCube 4-year point-source sample. The magnetic deflections of CRs have to be accounted for in the likelihood tests. For simplicity, we model individual deflections as a random variable 2-dimensional Gaussian distribution with the energy dependent standard deviation $\sigma_{M D}\left(E_{C R}\right)=\mathrm{D} \times 100 \mathrm{EeV} / E_{C R}$, and we consider the representative values $\mathrm{D}=3^{\circ}, 6^{\circ}$ and $9^{\circ}$ (the latter is just used for the likelihood test with the high-energy cascades and high-energy tracks). These values are reasonable test values as shown by a backtracking simulation of the detected UHECRs in the galactic magnetic field models of Pshirkov et al. [12] and Jansson and Farrar [13], presented in [14].

\subsection{UHECR correlation searches with high-energy cascades and tracks}

The arrival directions of the high-energy tracks and cascades in IceCube, and of the UHECRs measured by Auger and TA are shown in Fig. 1 in Galactic coordinates. Two different analyses are performed with these data sets: a cross-correlation and a stacking likelihood analysis.

Cross-correlation analyses: The cross-correlation method consists of computing the number of UHECR- $v$ pairs as a function of their angular separation $\alpha, n_{p}(\alpha)$, and comparing it to the expectation from an isotropic distribution of arrival directions of CRs. The angular scan performed in this case is between $1^{\circ}$ and $30^{\circ}$ with a step of $1^{\circ}$ and, due to this scan, the method does not rely on any assumption about the exact value of the strength of the magnetic deflections, unlike the likelihood method.

Likelihood stacking analyses: Stacking a set of sources is a conventional way of enhancing the statistical weight of multiple weak signals to enhance the discovery potential. Since $v$ s are not deflected on their way to Earth, $v$ arrival directions are stacked. An unbinned likelihood method is used, with the 


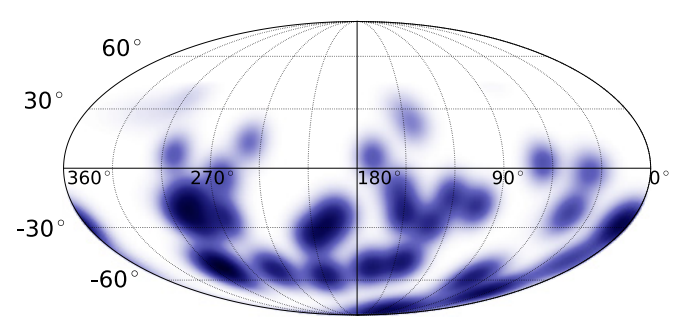

(a)

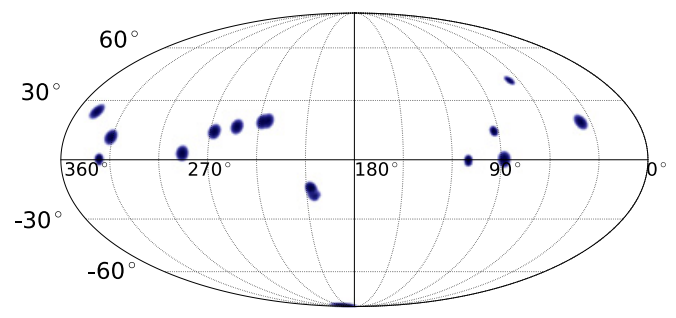

(b)

Figure 2. Neutrino likelihood maps convoluted with the declination dependent exposure of Auger in Equatorial coordinates. The plot on the left is for the high-energy cascades, while the one on the right is for the high-energy tracks. Similar figures for the exposures of TA can be seen in [14].
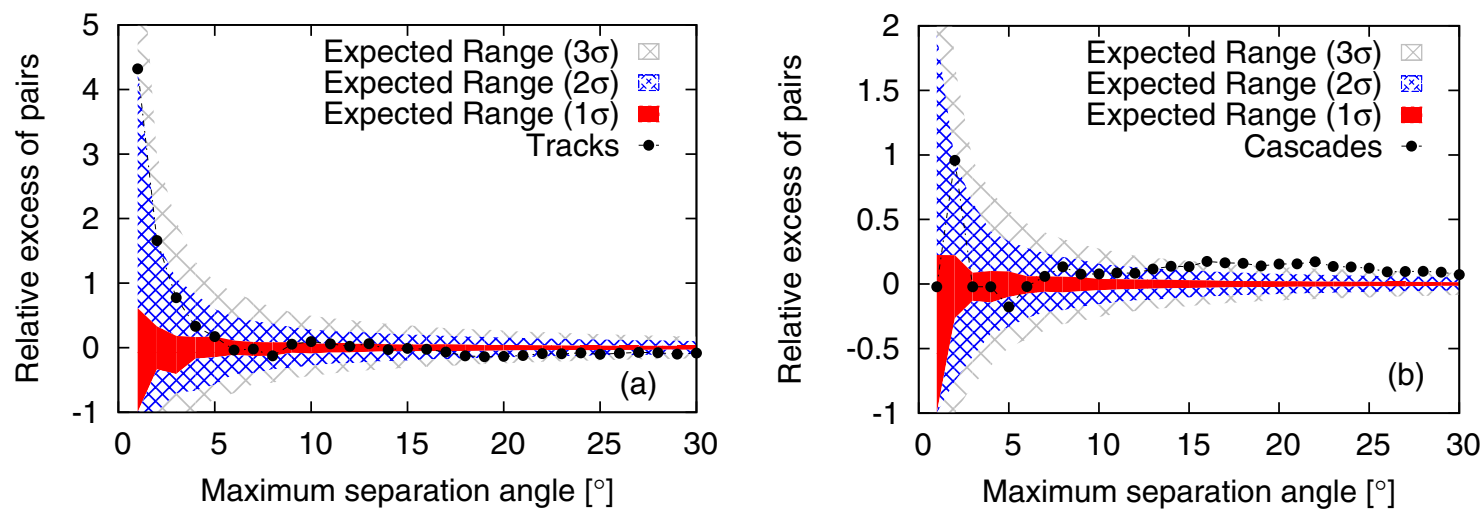

Figure 3. Relative excess of pairs, $\left[n_{\mathrm{p}}(\alpha) /\left\langle n_{\mathrm{p}}^{\text {iso }}(\alpha)\right\rangle\right]-1$, as a function of the maximum angular separation between the neutrino and UHECR pairs, for the analysis done with the track-like events (a) and with the cascade events (b). The $1 \sigma, 2 \sigma$ and $3 \sigma$ fluctuations expected from an isotropic distribution of arrival directions of CRs are shown in red, blue and grey, respectively.

$\log$ of the likelihood function defined as:

$$
\log \mathcal{L}\left(n_{\mathrm{s}}\right)=\sum_{i=1}^{N_{\text {Auger }}} \log \left(\frac{n_{\mathrm{s}}}{N_{\mathrm{CR}}} \mathcal{S}_{\text {Auger }}^{i}+\frac{N_{\mathrm{CR}}-n_{\mathrm{s}}}{N_{\mathrm{CR}}} \mathcal{B}_{\text {Auger }}^{i}\right)+\sum_{i=1}^{N_{\mathrm{TA}}} \log \left(\frac{n_{\mathrm{s}}}{N_{\mathrm{CR}}} \mathcal{S}_{\mathrm{TA}}^{i}+\frac{N_{\mathrm{CR}}-n_{\mathrm{s}}}{N_{\mathrm{CR}}} \mathcal{B}_{\mathrm{TA}}^{i}\right),
$$

where $n_{\mathrm{s}}$, the number of signal events, is the only free parameter and $N_{\mathrm{CR}}=N_{\text {Auger }}+N_{\mathrm{TA}}$ is the total number of UHECRs. $S_{\text {Auger }}^{i}$ and $S_{\mathrm{TA}}^{i}$ are the signal probability density functions (PDFs) for Auger and TA respectively and are Gaussians centered at the arrival directions of the UHECRs with widths accounting for their magnetic deflection and angular uncertainty [14]. $B_{\text {Auger }}^{i}$ and $B_{\mathrm{TA}}^{i}$ are the corresponding background PDFs representing the probabilities of observing a CR from a given direction assuming an isotropic flux. Consequently they are taken to be the Auger and TA normalized exposures. The log-likelihood of Eq. (1) is maximized with respect to $n_{\mathrm{S}}$ and the ratio of the maximum likelihood w.r.t the case where $n_{\mathrm{s}}=0$ is taken as the test statistic [14].

Figure 2 shows the normalized neutrino likelihood maps convoluted with the exposure of Auger to demonstrate the spread of the different observed neutrino arrival directions visible from the site.

Results: In Fig. 3 we show the results obtained upon applying the cross-correlation method to the data. For the case of the sample of high-energy tracks, the maximum departure from the isotropic 
expectation of CRs (fixing the positions of the $v \mathrm{~s}$ ) is at an angular distance of $1^{\circ}$, where 0.38 pairs are expected on average and 2 pairs are detected. The post-trial $p$-value is $28 \%$. For the analysis done using the high-energy cascade events, the smallest pre-trial $p$-value occurs at an angular distance of $22^{\circ}$, for which 575 pairs are observed while 490.3 are expected on average. The post-trial $p$-value is $5 \times 10^{-4}$ with respect to expectations of an isotropic flux of CRs. As an a posteriori study, we also evaluated the significance under the hypothesis of an isotropic distribution of neutrinos, fixing the UHECR arrival directions (note that this alternative hypothesis preserves the degree of anisotropy in the arrival directions of CRs that is suggested by the TA "hot spot" [15] or the excess around Cen A reported by Auger [7]). The post-trial $p$-value is $8.5 \times 10^{-3}$. For the likelihood stacking analysis, the most significant deviation from the isotropic flux is found for the magnetic deflection parameter $D=6^{\circ}$ for the cascade sample. The observed pre-trial $p$-value is $2.7 \times 10^{-4}$. Using a conservative method to estimate the trial factor [14], the post-trial $p$-value is found to be $8.0 \times 10^{-4}$. For this search the posttrial $p$-value with respect to the isotropic neutrino flux hypothesis is $1.3 \times 10^{-3}(\sim 3 \sigma)$. We see that for both the cross-correlation and the likelihood stacking analyses, the $p$-values obtained under the null hypothesis of isotropic neutrinos turn out to be larger than the ones obtained under the null hypothesis of isotropic CRs, the differences reflecting the extent to which the original $p$-values, from the isotropic cosmic-ray hypothesis, are due to an accidental alignment of the neutrinos with the known clustering of the cosmic rays.

\subsection{Stacking search for neutrino point-sources in the 4 year point-source sample}

The $v$ data set used for this analysis is the IceCube point-source data set. A stacking analysis is done but in this case (as opposed to the previous one) the stacked sources are the measured positions of UHECRs. An unbinned likelihood method is performed where the log likelihood is defined as:

$$
\log \mathcal{L}\left(n_{\mathrm{s}}, \gamma\right)=\sum_{i=1}^{N_{v}} \log \left(\frac{n_{\mathrm{s}}}{N_{\nu}} \mathcal{S}_{i}^{\text {tot }}+\left(1-\frac{n_{\mathrm{s}}}{N_{\nu}}\right) \mathcal{B}_{i}\right),
$$

where $n_{\mathrm{s}}$ is the number of signal events in the sample and $\gamma$ is the spectral index of the neutrino source candidates, assumed to collectively follow an unbroken power-law spectrum $\propto E^{-\gamma} . N_{\nu}$ is the total number of astrophysical neutrino candidate events in the sample. $\mathcal{S}_{i}^{\text {tot }}$ is the signal PDF for the stacked sources and $\mathcal{B}_{i}$ is the background PDF. The $\log$-likelihood, $\log (\mathcal{L})$ is maximized w.r.t. $n_{\mathrm{s}}$ and $\gamma$ and the TS is defined as in Sect. 3.1.

Only UHECRs above a certain energy $E_{t h}=85 \mathrm{EeV}$ have been considered. The value of $E_{t h}$ was decided using the procedure described in [14].

Applying the method to the actual data, all observations are found to be compatible with the background only hypothesis. The smallest post-trial $p$-value is $25 \%$ for the hypothesis of $D=3^{\circ}$, with a fitted excess of $\sim 123$ events and $\gamma=-3.24$. The analysis with $D=6^{\circ}$ yields a $p$-value larger than $50 \%$.

\section{Conclusions}

Three analyses have been performed to investigate correlations between UHECRs detected by the Pierre Auger Observatory and Telescope Array with various samples of IceCube $v$ candidates. The results obtained are all below $3.3 \sigma$. There is a potentially interesting result in the analyses performed with the set of high-energy cascades when compared to assumed isotropic arrival directions of CRs. If we compare the result to an isotropic flux of neutrinos (fixing the positions of the CRs) to consider the effect of anisotropies in the arrival directions of CRs (such as the TA hot spot), the significance is $\sim 2.4 \sigma$. These results were obtained with relatively few events and we will update these analyses in future with increasing statistics to follow their evolution. 


\section{References}

[1] A. Achterberg et al. [IceCube Coll.] Astropart. Phys. 26, 155 (2006)

[2] M.G. Aartsen et al. [The IceCube Collaboration], Proc. of the $34^{\text {th }}$ Int. Cosmic Ray Conf. 1081 (2015)

[3] M.G. Aartsen et al. [The IceCube Collaboration], Phys. Rev. Lett. 115(8), 081102 (2015)

[4] M.G. Aartsen et al. [The IceCube Collaboration], Astrophys. J. 796, 109 (2014)

[5] J. Abraham et al. [The Pierre Auger Collaboration] Nucl. Instrum. Meth. A523, 50 (2004)

[6] A. Aab et al. [The Pierre Auger Collaboration] Nucl. Instrum. Meth. A 798, 172 (2015)

[7] A. Aab et al. [The Pierre Auger Collaboration], Astrophys. J. 804, 1 (2015)

[8] T. Abu-Zayyad et al. [Telescope Array Collaboration], Nucl. Instrum. Meth. A 689, 87 (2012)

[9] T. Abu-Zayyad et al. [The Telescope Array Collaboration], Astrophys. J. 777, 88 (2013)

[10] J. Abraham et al. [The Pierre Auger Collaboration], Phys. Rev. Lett. 101, 061101 (2008)

[11] T. Abu-Zayyad et al. [The Telescope Array Collaboration], Astrophys. J. 768, L1 (2013)

[12] Pshirkov M.S., Tinyakov P.G., Kronberg P.P., Newton-McGee K.J., Astrophys. J., 738, 192, 10.1088/0004-637X/738/2/192

[13] R. Jansson, G.R. Farrar, Astrophys. J. 757, 14 (2012)

[14] M.G. Aartsen et al. [The IceCube, Pierre Auger and Telescope Array Collaborations], Proc. of the $34^{\text {th }}$ Int. Cosmic Ray Conf. 1082 (2015)

[15] R.U. Abbasi et al. [The Telescope Array Collaboration], Astrophys. J. 790, L21 (2014) 